\title{
Enhancing Space Exploration by Adding Astrosociology to the STEM Model
}

\author{
Jim Pass* \\ Astrosociology.com, Huntington Beach, CA, 92647
}

\begin{abstract}
[Abstract]. The STEM acronym emphasizes the renewed desire by space professionals (including NASA) and others involved in education to focus more strongly on science, technology, engineering, and mathematics. Based on the precipitous decline in the number of students choosing these areas for their future careers, this emphasis seems reasonable. NASA, as the example utilized in this paper, faces a dearth of qualified people in the workforce to replace their aging employee base (as do aerospace corporations). One major problem exists with this effort, however. While it seems as though this approach attempts to improve the composition of the workforce to one favorable to NASA's future hiring practices, it only partially accomplishes this objective. If the future includes a trend in which a growing number of people go into space to work and even live their lives, then STEM suffers from a missing component. The social sciences (which include the behavioral sciences and humanities for the purpose of brevity) will become increasingly important for reasons discussed in this article. Astrosociology consists mainly of social scientists interested in conducting outer space research. It concentrates on psychological, social, and cultural patterns related to space. Astrosociology adds a missing perspective to the space community that can no longer advance without integrating its core concepts, principles, and empirical findings to its future planning. For the space community, the emphasis designated by STEM requires change to STEMA to reflect the addition of astrosociology. This article makes the general case for bringing the social sciences into the space age, and into the space community, in a formal and permanent manner for the benefit of society and space exploration.
\end{abstract}

\section{Introduction}

$\mathrm{F}$ UNDAMENTALLY, this article addresses the various issues associated with realizing the goal of bringing outer space into the social science classroom. ${ }^{1}$ It calls for, and provides arguments to support, extending the emphasis on STEM subjects (i.e., those closely related to the natural sciences, technology, engineering, and mathematics) to include the social sciences by involving students whom are motivated by space and wish to pursue the study of space issues in their academic careers. In this case, rather than becoming the traditional engineers or planetary scientists, for example, students study to become astrosociologists who may specialize in a whole host of different subject areas that tie humanity as a social species to outer space (see Table Two). A major assumption here involves the idea that human beings will move beyond low Earth orbit relatively soon and do so as members of long-term missions and even permanent settlements. This reality will necessitate the training of experts in both the natural sciences and the social sciences to cope with this new growing need to accommodate people. The need to replicate human societies in space serves as an ideal illustration of both the limits of STEM education and the benefits related to combining it with a social-scientific perspective - specifically, astrosociology. Space societies will require successful implementations of both physical and social environments in order to become inhabitable. ${ }^{2}$ The acronym "STEMA" includes an astrosociology component, a new model that can increase interest levels in social science general education and train potential astrosociologists.

Consequently, even during a period in which NASA is focusing on human spaceflight, and shifting funds from its science programs to pay for it, the agency should nevertheless fund a modest new program to focus on an education program targeting social science students. While this proposal seems to defy both the historical avoidance of the social sciences in favor of a budget orientation that overwhelmingly favors the natural sciences, a small allocation for social science programs will prove timely and invaluable. NASA, the National Science Foundation

${ }^{*}$ Founder, Field of Astrosociology and Astrosociology.com, P.O. Box 1129, Huntington Beach, CA 92647, Member. 
(NSF), and other funding sources should provide support for at even modest programs that bring space into social science classrooms. The social sciences should receive representation in the guise of the new field of astrosociology as this field has developed further by far than any other social-science oriented field focusing on outer space.

In part, the insertion of astrosociology into courses and programs approximates expanding what is currently known as human factors so as to involve a vast array of concepts, theories, and research findings common to social scientists and adaptable to space environments. Beyond assistance from entities such as NASA and the NSF, educational programs at all institutional levels should also embrace space as a motivational tool for learning in social studies and social science classes on their own. This could serve as a first step toward establishing astrosociology programs in our schools. Astronomy programs would also benefit from an astrosociology course oriented toward the social and cultural relevance of conducting astronomical research.

\section{A. Definition and Relevance of Astrosociology}

Astrosociology, first coined in 1995 by Allen Tough ${ }^{3}$ and founded as a proper field in 2003 by the author, is defined as the study of astrosocial phenomena (i.e., social and cultural patterns related to outer space). It is simultaneously a sociological subfield and multidisciplinary social science field. As a sociological subfield, it includes in its purview all areas of research and theory that ties human behavior to space at all levels of social analysis from the micro level (involving social interaction among two or just a few people) to the macro level (involving large-scale patterns, such as human migration into space, and society as a major form of social structure).

As a proper multidisciplinary field, astrosociology includes each of the social and behavioral sciences, the humanities, and the arts. The concentration on space binds these diverse disciplines together to fill a neglected niche that complements the space community's traditional concentration on the natural sciences. It finally places an emphasis on the numerous complications associated with the long-neglected human dimension. ${ }^{4}$ To state the obvious, human space exploration will inevitable involve human beings! Stated another way, "spacefaring is a partnership between technology and people" [emphasis added]. ${ }^{5}$ And while the recognition of the human dimension sheds light on a long-overlooked list of social science issues, it also places a new burden on planners of space missions due to the social, psychological, and cultural complexities added to the traditional human factors approach (which is less diverse or complex by comparison). The new field of astrosociology was established to focus on these types of issues. A proper focus on the human dimension is by no means a new idea. However, the establishment of a formal field to deal with it formally is indeed a new form of progress.

Astrosociology also includes a practical component called applied astrosociology in which knowledge gained from astrosociological research is utilized in a "hands on" manner. The input of social scientists will increase in importance as humanity ventures into the cosmos. For example, the establishment and sustainability of space societies (i.e., space settlements) involves sociological, psychological, and psychosocial factors that complement the work of space engineers and architects. After all, a space society exists for the benefit of the human beings who live in them (rather than the engineers who build them). People should be part of the equation from the beginning of planning rather than comprising a mere afterthought once they are "dropped off" at the habitat.

The relevance of astrosociology resides in its focus on the human dimension. The need to accommodate human needs and desires adds a great deal of complexity and uncertainty to space exploration research. Engineers and architects will need to design their spacecraft and habitats to keep human beings alive, and moreover, to allow for a decent standard of living. Again, the social environment represents a complementary dimension to the commonly focused upon physical environment. Furthermore, astrosociology focuses on human behavioral patterns related to space exploration, astrosocial phenomena, whether they occur on Earth or in space. As such, a different type of scientist will be required to work alongside traditional members of the space community. Otherwise, expansion of humanity into outer space will become increasingly difficult and misunderstood.

\section{Assessment of STEM Education in the United States}

The shortage of qualified workers in the STEM subjects, whenever it occurs, certainly creates a problematic situation for a post-industrial society such as the United States. In the context of this article, it arguably affects the availability of qualified workers in space and aerospace organizations and weakens the security of the United States. ${ }^{6}$ Until we solve this current problem within the population of the United States, qualified workers will need to enter this country from international programs to avoid a decline in the progress of space technology and science. The U.S. will also need to minimize educating foreign students who take their new knowledge and skills back with them to their country of origin. These graduates need to remain in the U.S. if incentives can help ensure such as outcome. A decrease in STEM graduates will certainly translate into a decline in the rate of overall technological 
and scientific advancement; something that many logically characterize as intolerable. In fact, a post-industrial nation is characterized by its strong commitment to STEM-related subjects and research.

As indicated in Table 1 below, the United States is currently far below its once leading position as the top STEM degree production among nations. This table includes countries that award more than 200,000 university degrees on an annual basis. In fact, among these nations, the United States is the lowest producer of STEM degrees except for Brazil. "Although the U.S. ranks near the bottom in the proportion of STEM degrees, it ranks third (behind Japan and China) in the absolute number of STEM degrees awarded." "This last fact is devastating if it represents the incipient stage of a long-term period of decline. Further, the cooperation of European nations represents a total number of degrees that vastly dwarfs that of the United States.

Another question that bears consideration is whether the percentage of STEM degrees $(16.8 \%$ for the United States) necessarily represents a negative situation. While it is not good in the purest sense (from the perspective that we will become deficient in the number of STEM graduates in the near future), this article argues that it may not be totally negative in the area of space research if astrosociology is allowed to develop within American educational organizations (i.e., schools). Astrosociology, in a sense, demonstrates that the STEM subjects, while extremely important, cannot contend with every area of study that ultimately becomes vital to carrying out space exploration successfully. Even so, any post-industrial nation requires a "healthy" number of STEM graduates.

\begin{tabular}{|l|r|r|r|}
\hline \multicolumn{3}{|c|}{ Table 1. Field of First University Degree, by Selected Region and Country, } \\
2002 or the Most Recent Year Available \\
\hline Region/Country & All fields & STEM Fields & Percent STEM \\
\hline All regions & $9,057,193$ & $2,395,238$ & $26.4 \%$ \\
\hline Asia & $3,224,593$ & $1,073,369$ & $33.3 \%$ \\
\hline China (2003) & 929,598 & 484,704 & $52.1 \%$ \\
\hline India (1990) & 750,000 & 176,036 & $23.5 \%$ \\
\hline Japan (2004) & 548,897 & 351,299 & $64.0 \%$ \\
\hline South Korea & 239,793 & 97,307 & $40.6 \%$ \\
\hline Middle East & 445,488 & 104,974 & $23.6 \%$ \\
\hline Europe & $2,682,448$ & 713,274 & $26.6 \%$ \\
\hline France & 309,009 & 83,984 & $27.2 \%$ \\
\hline Spain & 211,979 & 55,418 & $26.1 \%$ \\
\hline United Kingdom (2003) & 282,380 & 72,810 & $25.8 \%$ \\
\hline Central/Eastern Europe & $1,176,898$ & 319,188 & $27.1 \%$ \\
\hline Russia (1999) & 554,814 & 183,729 & $33.1 \%$ \\
\hline North/Central America & $1,827,226$ & 341,526 & $18.7 \%$ \\
\hline Mexico & 321,799 & 80,315 & $25.0 \%$ \\
\hline United States & $1,305,730$ & 219,175 & $16.8 \%$ \\
\hline South America & 543,805 & 96,724 & $17.8 \%$ \\
\hline Brazil (2001) & 395,988 & 61,281 & $15.5 \%$ \\
\hline Source: National Science Foundation, Science and Engineering Indicators, 2006, Volume 1, Arlington, \\
\hline VA, NSB 06-01, January 2006.
\end{tabular}

Table 1 demonstrates that little doubt exists that the United States needs to take concerted efforts to increase the sheer numbers of STEM degree recipients within its population. In this vein, it is indeed important for the future of the U.S. to institute successful programs that motivate students to pursue STEM subjects in their classrooms. At the same time, this argument involves the increase in graduates from current levels. It does not realistically call for every student to pursue STEM subjects (nor should it). That is, the best outcome for the proper study of space exploration issues involves an healthy increase in STEM graduates and the new presence of astrosociology graduates and their increase of time. Astrosociology is not a threat to STEM education. It is an augmentation.

This article deals specifically with STEM education as it relates to improving research associated with space exploration. This focus involves a consideration of astrosociology as both an alternative choice by students but also an enhancement to the current approach. Space exploration promises to enhance the probability of such an outcome. 
NASA became involved in STEM education efforts because a reduction in STEM-related graduates poses a future hardship on the agency and because it represented the government's entity focusing on space exploration. The general idea is that bringing examples and exercises tied to space into STEM-related classrooms will motivate students who would otherwise show little or no interest in these subjects to pursue them as their areas of study and as majors at the college/university level. If space exploration can, in fact, motivate STEM students, it should be capable of motivating social science students to pursue astrosociological specializations. For the sociology and other social science programs, astrosociology may increase their student rolls from current levels.

Thus, we must go even further than STEM for the benefit of the United States. We must also pursue utilizing the motivation that space exploration brings in social science classrooms. Making connections between space and the social sciences, the fundamental purpose of astrosociology, will result in the development of well-qualified astrosociologists - an outcome just as important as STEM graduates if one accepts the premise that humanity will migrate to a significant extent beyond the Earth. Astrosociology must be added to the STEM concept for the sake of future progress both in space and on terrestrial soil. The renewed emphasis on STEM subjects and their relationship to our understanding of space exploration and related topics is important, yet this approach is unidimensional. It is unsustainable to ensure the success of human beings in space environments or to study the effects of space on terrestrial societies because these effects involve astrosocial phenomena, the very focus of astrosociology. As the missing perspective, astrosociology will add new insights to the prevailing ideas in the space community. In the process, new fundamental areas of knowledge will begin to take shape based on a formal and ongoing collaboration between the two branches of science. In order to make this possible, we need to educate and train both STEMoriented students and astrosociologists.

\section{A. Implications of NASA Impact on STEM Students}

In order to test the hypothesis that "bringing outer space in the classroom will encourage social science students to pursue astrosociology," one must begin with STEM students. The use of outer space to motivate STEM students to focus on STEM subjects falls under the direction of NASA's Office of Education. This office runs several programs in this regard including Explorer Schools, Educator Astronauts, internships and scholarships. NASA has a well-conceived plan for moving STEM students through their educational careers and into both the NASA and more general aerospace workforces.

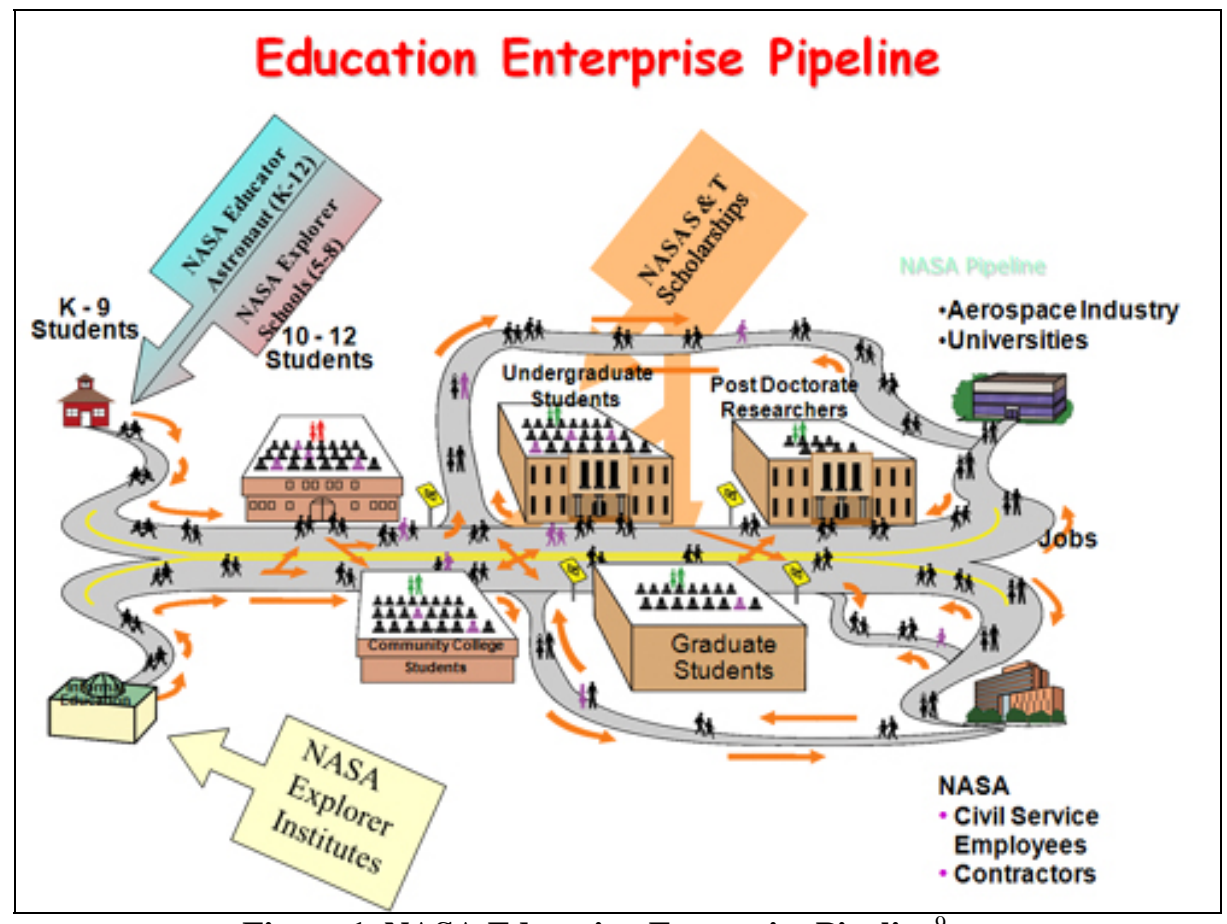

Figure 1. NASA Education Enterprise Pipeline ${ }^{9}$

The plan depicted in Figure 1 revolves around seeding students at the various levels of education so as to allow them to maximize their potential success and retain a high motivational level to join the aerospace industry 
workforce once they graduate from their universities. It starts in kindergarten and continues on into college. And while NASA promotes space in a limited number of classrooms, the idea is to demonstrate the success of this model so that other schools implement similar programs on their own.

NASA uses various metrics in the attempt to gauge the success level of its educational programs. Figure 2 indicates definite improvement among students in STEM classrooms after exposure to NASA programs. These increases validate the idea that bringing space into the classroom can make a difference.

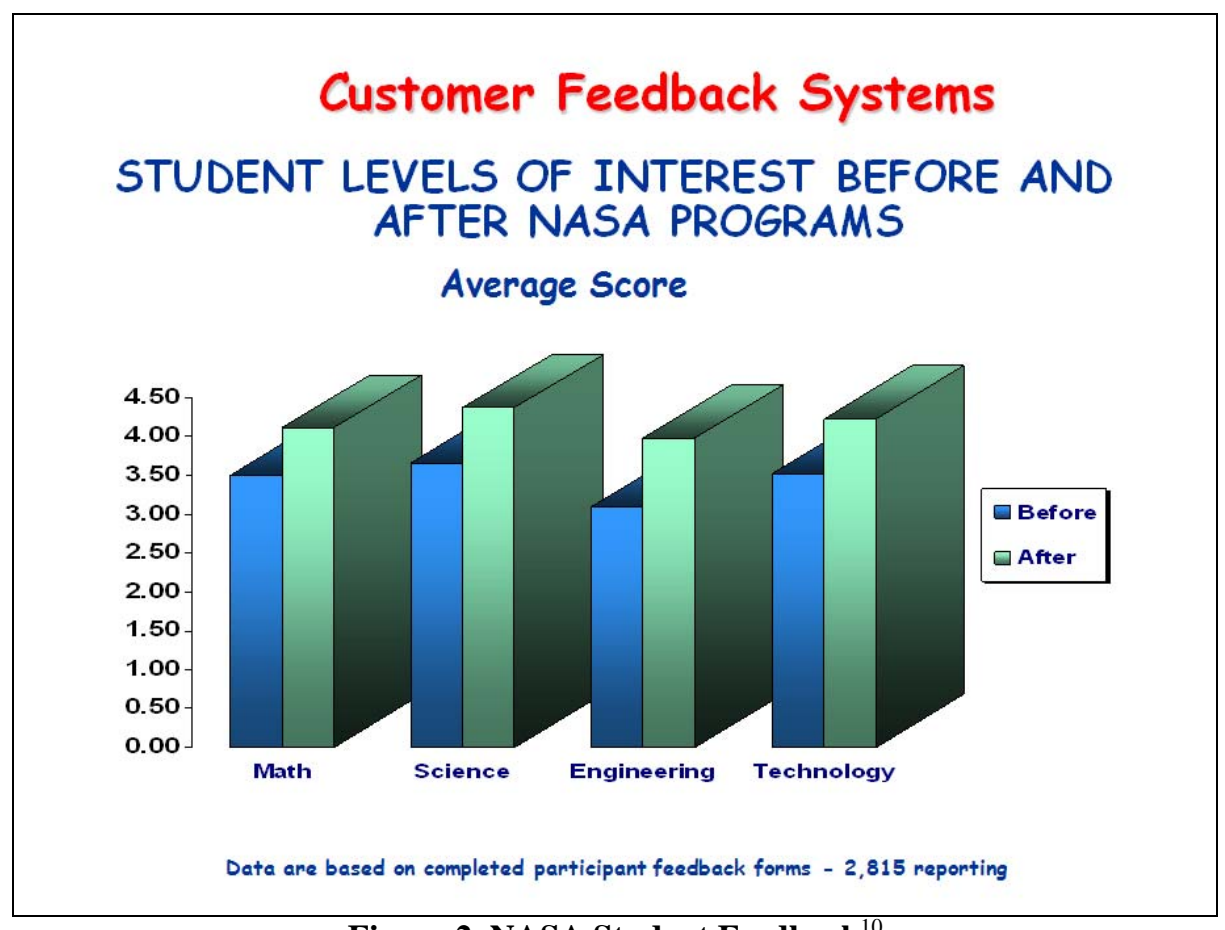

Figure 2. NASA Student Feedback ${ }^{10}$

NASA utilizes four different types of methods to evaluate their programs. They include (1) program assessment, (2) metrics collection, (3) program evaluation, and (4) program oversight. ${ }^{11}$ The specific measurement tools may change over time as NASA improves its methods so as to enhance the effectiveness of its programs. The focus on STEM subjects is prevalent in the NASA literature. The social sciences receive much less attention.

However, what about students who favor a social science education? How would a similar exposure to spaceoriented programs affect students who favor the social sciences? One can imagine that an increase would occur due to a general interest in space indicated in the general population and especially among children in addition to its novelty in their typical curriculum. On the other hand, students may find it uninteresting due to its traditional separation from social science education. Regarding this second scenario, it may take some convincing to demonstrate that a social science approach to space exploration is truly sociology, for example, rather than disguised engineering or geology. Evoking the space settlement example once more, it should become clear to these students relatively quickly that the same core subfields in sociology (e.g., deviance, family, medical sociology, racism, sexism, political economy, religion, and social class) will remain highly relevant in social environments located off humanity's home planet. Moreover, applied astrosociology will allow them to participate in planning the details, and monitoring the functioning, of space societies.

\section{Subjects Relevant to Astrosociology}

Before examining the pedagogical merit of astrosociology and related issues, it must be demonstrated - even if briefly - that this new field is important enough to develop for both the space and social science communities as well as to benefit societies in general. It would make no sense to add this new field to current academic programs if it possessed no intrinsic worth as a subject matter or if it duplicated existing disciplines or fields (the latter point to be examined in the following paragraph). As it happens, it is quite a simple matter to demonstrate the relevance of astrosociology to human social life - in past, present, and most notably future context. A topic relevant to astrosociology is, by definition, also relevant to terrestrial human societies because some element of it will always 
focus on the relationship between outer space and society. Currently, of course, the only existing human societies reside on the Earth's surface.

Duplication of existing programs and departments will occur at first to a limited extent as some space-oriented programs already exist. Those who run such programs as "space studies" or "space policy" will need to decide whether they wish to remain independent or join the banner of "astrosociology." To the extent the latter occurs, astrosociology would become a mainstream subject area within academia that much more quickly. For nearly all traditional sociology and other social/behavioral science programs, however, astrosociology would represent a highly unique subject area. A growth in the popularity of astrosociology would pressure department chairs, faculty, and administrators to consider adding this new subject in the form of courses and eventually programs that allow students to minor or major in astrosociology. The focus on space issues remains so uncommon in social science departments that duplication is much less of an issue than the sheer effort and cost of implementing the new program as a new focus for the great majority of them.

Thus, even though we are only fifty years into the space age, the subjects relevant to both astrosociology and society consist of those listed in Table 2 as well as many others the author has listed. ${ }^{12}$ These subject areas represent some of the core specializations within astrosociology, and they demonstrate the contributions this new field will add to current research and understanding within the space community as it has traditionally functioned in the past. In most cases, the astrosociological perspective provides a broadening of the conventional approach common to the natural and physical sciences. In effect, it adds the human dimension to the current orientation that focuses most strongly on STEM subjects which tend to involve nonhuman systems. Astrosociology allows for the study of human systems to be added and emphasizes the integration of the two types of systems. One may think of it as human factors on steroids in the sense that the experts in human behavior - that is, those who train in the social sciences utilize their theoretical and observational skills in the study of astrosocial phenomena and related concepts.

Each of the subjects in Table 2 represents a potential specialization under the astrosociology umbrella. From the social science perspective, astrosociology consists of specializations that are both new to, and already present in, various existing programs. Potentially, each subject area could develop eventually into a specialized major or minor within an astrosociology program within a social science department. These topics are provided to demonstrate the variety of concentrations possible. Some are already common in existing programs - such as space societies, space law, and space policy, while others - such as spacefaring society, planetary defense, and medical astrosociology are not common at all. The International Space University (ISU) is a unique program that encompasses many of these subject areas though not under the guise of "astrosociology." ${ }^{\dagger}$ For example, departments exist called the "Space and Society Department," the "Space Policy and Law Department," and the "Space Business and Management Department." In the future, the astrosociology movement will push for universities to change their existing names to the "Astrosociology Department" or astrosociology to their curricula in ways that are consistent with their existing organizational structures.

Another good example of the potential synergy gained by combining the approaches of the space community and the social science community involves the study of astrobiology including the Search for Extraterrestrial Intelligence (SETI). The social, cultural, and psychological ramifications of discovering alien life are too numerous to discuss here, but they would change humanity forever in a great variety of ways. Even the search itself possesses characteristics worthy of study. Past research on this general topic by social scientists does indeed exist, though one cannot characterize this body of work as a well-structured body of knowledge. By placing it under the purview of astrosociology as a formal specialization, the location of this research would be more accessible and the ability to contribute would be enhanced. Similar improvements would occur with the other subjects proposed as astrosociological specializations.

Astrosociology would also possess relevance as a course in astronomy programs because students would learn about the various connections between human beings and space. This requires specialized education, then, and therefore the further development of astrosociology possesses pedagogical significance for non-social science departments and programs. The fact that it is currently unavailable in astronomy programs across the United States indicates the persistence of the status quo. The STEMA movement seeks to change this reality. Astronomy professors and instructors could access astrosociological research relevant to their courses once astrosociology becomes more accepted in academia. The connection between human cultures and the cosmos are important as astronomy always occurs in a human context and societies are somewhat shaped by theories and discoveries. Moreover, many of these faculty members would probably become motivated to conduct their own research and thereby contribute to the literature regarding "space and human cultures." This serves as another excellent example

\footnotetext{
† The URL for the International Space University is as follows: http://www.isunet.edu/
} 
of a good synergy between the social sciences and the natural sciences. Such a specialization could well develop into a course within astronomy departments.

\begin{tabular}{|c|c|}
\hline Astrosociological Specialization & Description \\
\hline Applied astrosociology & $\begin{array}{l}\text { Use of astrosociological knowledge to enhance planning of space } \\
\text { missions and projects in conjunction with traditional members of } \\
\text { the space community - primarily applicable to human spaceflight. }\end{array}$ \\
\hline Astrobiology / SETI & $\begin{array}{l}\text { Study of the relationship between human and extraterrestrial life; } \\
\text { including a focus on (1) the varying reactions to the discovery of } \\
\text { an unrelated genesis of life and (2) the very need to search. }\end{array}$ \\
\hline Medical astrosociology & $\begin{array}{l}\text { Space medicine with a concentration on social, cultural, ethical } \\
\text { issues and others in the tradition of medical sociology. }\end{array}$ \\
\hline Planetary defense & $\begin{array}{l}\text { Study of efforts taken to protect Earth, but also the protection of } \\
\text { societies and their cultures. }\end{array}$ \\
\hline Space law & $\begin{array}{l}\text { Determination of formal and informal norms regulating behavior } \\
\text { in space environments; including their purpose and consequences. }\end{array}$ \\
\hline Societal impact of space sciences & $\begin{array}{l}\text { Study of the impact of astrosocial phenomena on terrestrial } \\
\text { societies associated with the conduct of space scientists and the } \\
\text { reporting of their findings. }\end{array}$ \\
\hline Space policy & $\begin{array}{l}\text { Study of the decisions and action regarding the use outer space as } \\
\text { decided by societies; including cooperation and conflict in space. }\end{array}$ \\
\hline Social problems research & $\begin{array}{l}\text { A type of applied astrosociology that utilizes space assets to solve } \\
\text { and mitigate societal troubles in both terrestrial and } \\
\text { extraterrestrial environments. }\end{array}$ \\
\hline Space societies & $\begin{array}{l}\text { Study of issues associated with replicating human societies in } \\
\text { space, traditionally referred to as space settlements or colonies. }\end{array}$ \\
\hline Spacefaring society & $\begin{array}{l}\text { Prediction and identification of long-term trends toward a model } \\
\text { of society in which space becomes dominant in society and } \\
\text { culture (a developmental, potential outcome). }\end{array}$ \\
\hline
\end{tabular}

Before moving forward, it is important to recognize that the pioneers of astrosociology have a very difficult task ahead of them. Attempting to add an entirely new subfield of sociology or any social/behavioral science to an existing department is fraught with resistance and ridicule toward those who attempt it. Astrosociology itself must overcome suspicions of outsiders that it does not, in fact, represent a legitimate subject matter. Otherwise, why else are the equivalents of "astrosociology" programs not already part of social science departments? In all probability, astrosociology will need to overcome many of the legitimacy issues that programs related to the Search for Extraterrestrial Intelligence (SETI) fought and overcame during its development. ${ }^{13}$ Topics involving proving the existence of UFOs and alien abductions are often erroneously tied to astrosociology by critics who fail to understand the definition and purpose of this new field. Overcoming this misconception may indeed prove difficult.

\section{STEM Education at the Expense of the Social Sciences}

Having demonstrated the relevance of, and need for, astrosociology, we can now turn to issues related to the pedagogic potential of the field. Today, there exist no courses, programs, or departments dedicated formally to 
"astrosociology." Except for college courses taught by this author in the recent past, it remains probable that "astrosociology" is still never mentioned by name in sociology courses or any other social scientific courses. It seems exceedingly obvious to even mention it, but the proponents of astrosociology have much work ahead of them. At the same time, the successful establishment of astrosociology into educational organizations across the country, and indeed throughout the world, would prove most gratifying to supporters and edifying to current knowledge. Also quite obvious is the fact that tradition within the space community will prove somewhat difficult to change while the status quo of social science disciplines will prove much more resistive.

The danger related to the current drive within educational circles to improve on STEM education relates to the assessment among many educators that social science education is somehow less important - or even more extreme, irrelevant to the future of space exploration. It also implies that understanding human behavior and society represents a secondary status to understanding natural and physical phenomena. The pursuit of STEM education $a t$ the expense of the social sciences represents an extremely shortsighted and thus risky approach. The development of astrosociology counters such trends, though attitude favoring STEM subjects may also make it more difficult to develop this new field. Along with the development of astrosociology, then, advocates must constantly remind educators and policy makers of all stripes to maintain a proper balance between the natural sciences and the social sciences within the curricula of educational organizations at all levels. Within the space community, advocates should strive for a more equal balance although the space sciences and engineering fields will still dominate.

\section{A. The Status Quo and the Alternative}

By emphasizing the natural sciences, technology, engineering, and mathematics, NASA potentially alienates students interested in outer space but also partial to the social sciences. At the very least, this effort contributes to the prevailing belief (at the macro level) within social science disciplines and related fields that outer space has little to do with human behavior. In reality, space from a social science perspective is all about humankind. For example, space scientists do not study asteroids and comets just because they are out there. They study them in part because one may strike the Earth someday; one large enough to disrupt human societies potentially enough to knock them back to the Stone Age. Planetary defense is an important specialty within astrosociology as the protection of societies and their cultures is required. ${ }^{14}$ Moreover, findings from space scientists enrich the cultures of societies. Space policy is intertwined with social policy that affects a multitude of social institutions and groups. This social reality - the strong connection between outer space and society - requires addressing.

NASA's current approach infers that the social sciences are not very important to space exploration. This stance never was realistic throughout the space age, however. The social sciences tie efforts related to STEM and outer space to society. The irony is that an astrosociological approach represents an extremely relevant yet missing perspective. ${ }^{15}$ Thus, the best approach involves attracting students with interests in all subjects related to space exploration. The alternative to the status quo does not involve the replacement of long-standing tradition with something new. Rather, it involves the addition of astrosociology to the STEM subjects. Of great interest are the dynamic interactions between natural science students and social science students who both emphasize space as their primary interest. When this reaches a large enough scale, the potential outcomes of combined research between the two types of students will surpass what can emerge from strictly a natural science perspective. The same synergy will occur among professional scientists as well.

Now is not the time to discard the social sciences in favor of bolstering the STEM subjects and disciplines. Rather, a well-rounded approach is necessary, just as was always the case. We must not abandon the social sciences precisely because they represent an entire branch of science that comes under indirect attack by educational organizations (i.e., schools) that begin to emphasize science, technology, engineering, and mathematics over understanding human behavior as well, even as new complex patterns and trends will emerge as humanity increases the intensity of, and thus its reliance on, its connection to the cosmos.

Exposure to a well-rounded educational program provides students with exposure to fields and disciplines from the natural sciences, social and behavioral sciences, the humanities, and the arts. Such exposure fairly allows each student to select which field or discipline to pursue. Whenever a student opts to pursue anything related to space, whether a natural science or social science, the overall impact furthers space exploration. This outcome that includes the development of astrosociology possesses implications for improvements in science and technology as well as national security for a particular society. Moreover, the overall body of knowledge and its related literature improves. The traditional bifurcation between the study of outer space issues as a natural science privilege versus the inability to study outer space issues from a social science perspective makes little sense. STEM education at the expense of the social sciences no longer serves human societies well as we need both perspectives to carry out, and understand the effects of, the human exploration of space from Earth (e.g., astronomy or planetary defense) or in space environments (e.g., space settlement, long-duration spaceflight, or space tourism). 


\section{The Need to Expand from STEM to STEMA}

It is perfectly acceptable to utilize children's initial interest in outer space to motivate those who demonstrate both skills and interest in STEM subjects to pursue them as they progress through their levels of educational attainment. And the pursuit of bolstering the rolls of STEM students and successful graduates provides an answer to the dearth of students currently pursuing careers in science, technology, engineering, and mathematics. This problem requires a strong effort to solve. It seems clear that the Department of Education, NASA, and other organizations have responded to this problem in a big way.

In essence, however, this one-sided reaction involves limitations and fails to cater to all students. Not every student favors the STEM subjects nor has the aptitude to become successful in such courses. These students also require a good education to contribute to society in positive ways. Many students demonstrate skills and/or interests more appropriate to the social sciences. Based on earlier arguments, it is reasonable to hypothesize that many social science students would find inspiration and interest in an astrosociological approach to space research. Attempting to convert the social-science-oriented students into STEM-oriented students probably results in a waste of time. At the same time, without the possibility of pursuing astrosociology, their interest in space would most likely dissipate over time in favor of more traditional specializations within the social sciences.

Preparations for living, working, and recreating in outer space must involve a formal collaborative structure between the space community and the social science community. This will involve a new educational approach not now in existence: the formal training of astrosociologists. To accomplish this we must bring space into the social science classroom just as we do for STEM classrooms; that is, to add an "A" to the STEM acronym in the context of studying space exploration issues. Potentially, examples utilizing outer space inspires even those social science students who eventually go into other specialties by keeping them in school. It provides benefits for students beyond aspiring astrosociologists. On the other side of the ledger, an astrosociological perspective adds an exposure to concepts rarely discussed in either STEM-oriented or social science classes. When students learn about astrosocial phenomena, they gain a more comprehensive view of how space exploration relates to their everyday lives.

In addition, our society faces a growing need to study space exploration from a social science perspective. Rather than leaving students who turn down pursuit of the STEM subjects without options, we should motivate them by bringing the wonders of space exploration into the social science classroom. ${ }^{16}$ We need to assist those who wish to study the various specializations within the field of astrosociology. Is this important? Are there even enough students interested in space but from a social science perspective? These questions receive attention at this juncture. If astrosocial phenomena truly affect social life on Earth and humanity seeks to explore space by venturing out into it, then the relevance of astrosociology seems quite apparent.

\section{A. Summary of a Synergistic Effect}

By enhancing space exploration through the addition of astrosociology to the STEM approach to education, both the space community and the social sciences are enhanced. In fact, our overall society benefits as well on a general level. This is something that could not soon occur without the existence of astrosociology. The natural and social sciences comprise two sides of the same coin that represents human space exploration.

In short, then, "developing astrosociology for the space sciences" [and other avenues of research common in the space community] involves the addition of astrosocial knowledge to space-based knowledge to form a more balanced understanding of humanity's destiny in space. Knowledge relevant to the space sciences and engineering disciplines remain important, of course, but they represent only half of the overall knowledge base...Astrosocial knowledge represents the other side of a figurative coin. A great need exists to recognize each side of this figurative coin and bring both sides together to form the total knowledge needed to understand the full range of ramifications involving space and its important connections to terrestrial societies...This combination of both space knowledge and astrosocial knowledge possesses the potential to produce synergies of knowledge impossible by either knowledge base alone. Our continuing failure to pursue this avenue of research [i.e., astrosociological research] can only result in...[limited] progress, and perhaps avoidable negative circumstances. ${ }^{17}$

Thus far, we have concentrated heavily on the natural science side and largely neglected the social science side. We have overlooked the complementary aspect of space exploration in favor of concentrating on only one its two elements. Even when we send robotic probes, rovers, or landers to other worlds, we do so in order to further human knowledge. When we send human beings, we add innumerable complications that require input from social scientists that are fully prepared to cope with them.

The traditional pattern of concentrating on STEM subjects overlooks the rich history of sociology and the other social sciences. Countless research findings originally discovered during research aimed at terrestrial societies possess important applications toward social life in space environments that go far beyond the adaptation of findings associated with isolated social groups known as "space analogs." For example, when considering the space 
settlements, we must perceive it as the replication of societies in space that includes social groups and institutions as well as cultures. In fact, then, the natural scientists and the social scientists each need one another. ${ }^{18}$ The one-sided approach common to the past functioning of the space community will prove inadequate to move forward in the future if human beings are to move into space environments.

This overall discussion regarding the need to expose social science students to astrosociology suggests an important question. Are social science students even interested in the relationship between space exploration and society (or social/cultural issues)? After all, their ability to study such issues does not exist in today's educational system. Further, if there is no interest, then the entire argument of this article may represent a fruitless exercise. At best, it would indicate that extraordinary efforts may be required to interest social science students in space issues.

The pilot study conducted by the author could not address the specific interest in astrosociology among social science majors specifically. Instead, it surveys students who took an Introduction to Sociology class to fulfill their general education requirements - most of them were undeclared majors. But while this preliminary examination of the issue proved imperfect due to issues related to funding, access to students, and the types of students surveyed, it does indeed provide an enlightening glimpse into students' interest following a semester-long exposure to sociology.

\section{B. A Preliminary Survey of Sociology Students}

During my last three Introduction to Sociology courses at my most recent college, it turned out a fortunate circumstance that I decided to add an optional short answer item to the final exam of my last three classes before leaving the organization. Students had the option of answering this short-answer question or another concerning an aspect of social inequality. Because the proper development of astrosociology requires that it becomes part of curricula at a growing number of colleges and universities, it is important to gauge the interest in the new field among college freshmen and students at other levels of educational attainment.

1. Frequency Table

The results shown in Table 3 indicate a strong interest in astrosociology among an admittedly very small population (and thus sample) of general education college students. It is significant that these students were members of an Introduction to Sociology course rather than one that focused on space or technology in some way. This fact makes their interest in the "sociology of space exploration" all the more powerful as most of them were taking sociology to fill their general education requirements. Informally, most indicated to the author that they did not intend to pursue sociology.

\begin{tabular}{|c|c|c|c|c|}
\hline \multicolumn{5}{|c|}{ Table 3. Response Frequencies to Final Exam Extra-Credit Item ${ }^{\ddagger}$} \\
\hline Semester & $\begin{array}{c}\text { Interested in } \\
\text { Astrosociology }\end{array}$ & $\begin{array}{l}\text { Not interested in } \\
\text { Astrosociology }\end{array}$ & $\begin{array}{c}\text { Not interested in } \\
\text { Astrosociology, } \\
\text { But... }\end{array}$ & Totals \\
\hline $\begin{array}{l}\text { Spring } \\
2005\end{array}$ & $\begin{array}{c}13 \\
(29 \%)\end{array}$ & $\begin{array}{c}00 \\
(0 \%)\end{array}$ & $\begin{array}{c}04 \\
(09 \%)\end{array}$ & $\begin{array}{c}17 \\
(38 \%)\end{array}$ \\
\hline Fall 2005 & $\begin{array}{c}07 \\
(16 \%)\end{array}$ & $\begin{array}{c}02 \\
(04 \%)\end{array}$ & $\begin{array}{c}02 \\
(04 \%)\end{array}$ & $\begin{array}{c}11 \\
(24 \%)\end{array}$ \\
\hline $\begin{array}{l}\text { Spring } \\
2006\end{array}$ & $\begin{array}{c}16 \\
(36 \%)\end{array}$ & $\begin{array}{c}01 \\
(02 \%)\end{array}$ & $\begin{array}{c}00 \\
(0 \%)\end{array}$ & $\begin{array}{c}17 \\
(38 \%)\end{array}$ \\
\hline Totals & $\begin{array}{c}36 \\
(80 \%)\end{array}$ & $\begin{array}{c}03 \\
(07 \%)\end{array}$ & $\begin{array}{c}06 \\
(13 \%)\end{array}$ & $\begin{array}{c}45 \\
(100 \%)\end{array}$ \\
\hline $\begin{array}{l}\text { Note: the } \mathrm{F} \\
\text { total popul }\end{array}$ & $\begin{array}{l}\text { tages in each ce } \\
\text { size }(n=45) \text {. }\end{array}$ & resent the propo & of students in rel & to the \\
\hline
\end{tabular}

\footnotetext{
‡ The question on the three final exams read as follows: (2) Would you be interested in a course called Introduction to Astrosociology? This course would focus on issues associated with the relationship between space exploration and society, including the influences of space activities on social/cultural change and the future development of human societies on Earth and eventually in space itself. Please explain the reasons for your interest or lack of interest in astrosociology.
} 
The results in Table 3 mimic the general support of NASA's mission of space exploration found in the general population by major opinion polls, albeit even more strongly than those polls indicate. Potentially, then, many students who may pursue other subfields within social science disciplines would be attracted to astrosociology if it was available to them as a college course. While placing astrosociology in the curricula of academic programs at secondary and post-secondary levels will prove difficult, it will not necessarily prove impossible. One may infer that the example of astrobiology's development indicates a strong potential for astrosociology.

\section{Short Answers}

As evident by the examples of responses from students provided below, the breakdown into the three categories found in Table Two serves as a rather crude categorization scheme. Students who indicate they would not enroll in an astrosociology course tend to have mixed feelings about their decisions. The survey serves the purpose of an exploratory examination of the hypothesis that a significant number of social science students would enroll in an Introduction to Astrosociology course as part of their education (i.e., if the author taught such a class). Again, it does not assess whether or not they would pursue an astrosociology major if it were possible. This would require a much greater commitment and indicate a much greater level of support for astrosociology. The survey of sociology and other social science majors looms as an extremely important project for the future.

The five responses that follow are from students who clearly expressed an interest in astrosociology. They are representative of the positive responders (i.e., those "interested in astrosociology") in Table 3. In general, they express only positive sentiments.

The first student sees a bright future for astrosociology due to the impact of astrosocial phenomena.

I would be interested in Astrosociology because in the future as space exploration becomes more common and as space programs become more advanced, we will need to know its influences on society. We will need to know how the world will react to new discoveries in space like maybe new habitable planets or even new forms of life. The world is moving towards space exploration and the impact of it on the world can be studied by astrosociology. In the future, astrosociology will be more and more popular as it becomes evident that space exploration has a great impact on societies on Earth and can cause great social change.

The next student's positive response is also surprisingly insightful regarding the promise of astrosociology.

The opportunity to take an introductory class to astrosociology would be very ideal for me. As we grow in a technological would of advancement, especially in the United States, where we have achieved such greatness and accomplishments in the study of space exploration, the study of the solar system, the study of possible life outside our own world, and I hope of building communities on other planets, it is all wonderful and fascinating. The study of astrosociology would give us the knowledge and advanced opportunities to explore possible communities and resources outside our own planet. As it is, the world is experiencing overpopulation, as medical technology improves and life expectancy increases. Sociology is a wonderful and interesting subject that helps us to understand life and the interactions, functions and conflicts between/of people. By developing astrosociology as a subject that is just as credible, testable and available, we advance to another step to improving our lives on Earth and to understand life on an even "bigger picture.” There are so many factors that we can learn about; factors out in space that can affect life on Earth possibly in the near future. By learning about as much as we can about space exploration and society, we can better prepare ourselves for our future and future generations.

The respondent below provides a positive reaction based strongly on the idea that space exploration will become a growing dimension of social life as the future unfolds. The author has made similar arguments in the past.

Introduction to Astrosociology sounds really interesting. I would definitely be interested in this course. Just the name of the course catches a lot of my attention. In sociology, I learned about the problems we face in society, the challenges we can face later in life, and I would now be interested in what's going on out there. I think that my top reason for 
being interested in this course would be what changes can it bring us in the future, now, and can it affect society as a whole, as an individual, or both. I would also want to find out in what way it benefits human societies on Earth, and what kinds of developments on Earth will occur. With no doubt, I would take this class for further knowledge.

This student would take an astrosociology course due largely to the significant effects of astrosocial phenomena on contemporary society. The focus of this respondent is on social life on the planet Earth rather than humans in space - it is very insightful.

I personally would be interested in such a course simply because I have always been fascinated with space exploration and because I think there is a strong influence of space exploration on society. Take for example every space launch. It is covered by the media extensively and we are always interested in understanding our surroundings. It is also interesting to see the social consequences of successes or failures in space programs. I think many people dismiss the idea of space exploration and its influence on social/cultural change. However, if you really pay attention to how society reacts at everything NASA does, you begin to realize there is a definite connection.

The following student identifies an important aspect of astrosociology that many overlook. Astrosocial phenomena currently affect people, social groups, societies, and cultures on Earth much more than in space - and these effects demand proper attention!

I would definitely be interested in a course called Introduction to Astrosociology. I believe there is not much attention directed towards these studies and I would be highly interested in learning about the influences of space activities on social and cultural change because I do believe it plays a huge part in our development. As citizens, we have no idea yet. I would be interested in learning about this unique study and I believe it would benefit all to know more about the effects of space because it is such a huge contributor, none of us really think about it here on Earth how significant the effects of space really are.

The student above captures astrosociology's greatest contemporary impact on humanity. It is likely that most citizens simply do not recognize it.

The next four responses reflect students who did not find astrosociology altogether interesting. These students well represent those who fall in the "not interested in astrosociology, but..." category. They report mixed feelings to some extent. This type of response perhaps speaks to the idea that many people in the general population possess conflicting feelings about space exploration.

The next respondent sees greater value in studying social problems on Earth. The impact of space on terrestrial societies is not recognized.

I don't think I would be interested in the Astrosociology course because I think society on Earth is much more important than space activities. You say that this course would focus on the future development of human societies on Earth, but how can that happen if there are no humans living in space like there is here on Earth. I guess I just believe that we must fix the mess in our backyard before we go fix other yards. I don't think that Astrosociology would help me to better understand our society. I do think another sociology class will. On another note, if the class was free, I might take it just to see exactly what you will teach! (:)

Still, the student response above actually does indicate some curiosity at the end.

This next student possesses no interest in astrosociology, if the first sentence is any indication. For the most part, except for one sentence, the focus remains on the theme of learning about human behavior on Earth more thoroughly before venturing into space. 
If I'd be interested in Astrosociology: no. I work in the aerospace industry and do have an interest in space exploration to an extent. I don't think I believe in other life forms in space, but I do think the work of sociologists isn't done here on Earth. Perhaps a study of why people are so interested in life in outer space would interest me. If sociology spent more time learning about the diverse groups of people in their own communities, I think there would be more cohesiveness in the world, and a greater acceptance of that that is different.

Still, that one sentence within the overall response (displayed in red text) hinted at some interest after all. It does not fit in the "no interest" category because it does mention potential interest amidst a significant lack of enthusiasm. This respondent may or may not take the class, but would probably mention it to his or her friends. At this point, we cannot adequately assess the level of interest in the student population. However, even a conservative extrapolation from the current findings provides one with "cautious optimism” about astrosociology's future.

This next student may be confusing space science or natural science for social science. This is consistent with the status quo regarding space studies in which the STEM subjects receive greatest attention. There is a general assumption that space can only be understood from a natural science perspective. The student's curiosity may peak even more if he or she was informed more about the astrosociological approach as consisting of a sociological or social science orientation. Additionally, there is also a practical component to this student's hesitation to take an astrosociology course that may affect many community college students as well as freshmen and sophomores in general. They may not have the time or money to add extra classes to their course load and/or time in their personal schedules to do so. Finally, this student knows friends who would probably take the course.

I probably would not be interested in that course just because topics of that nature do not interest me. On the other hand, it does peak my curiosity. If I did not have a full time job and if I had more time on my hands, it may be worth a try. Still, normally topics such as Astrosociology do not spark anything for myself. I also tend to do very badly in classes of this topic. However, I do know from friends who do well in classes like this one and would probably love to check it out. If this class does open I could contact them if you want me to. You have my email address, so you can email me if it does.

The following student admits to an interest in astrosociology. However, this student mixes in concern for federal expenditures perhaps wasted on space exploration or "better spent in America today than on Mars." This particular student would probably take the course if it was practical vis-à-vis his or her schedule.

Actually, the correlation of these two subjects would be quite interesting. It has not been really discussed to an extent about how space exploration is going to affect society, especially in the future. What about going to Mars? I believe there definitely should be a look at the implications of these new types of space exploration. However, the money in President Bush's plan, anyway, would be better spent in America today than on Mars. There is millions upon millions of dollars in a deficit for the U.S.. I know that it is going to affect my taxes, interest rates, and social security in the future. The interest of science should be furthered, but not at the expense of human lives. My philosophy is basically live in the moment. Or in this case, fix the country now, not later. Mars can wait! See, I have already gone on about it so I would probably be interested in the sociological effects of astronomy.

The response above epitomizes the mixed character of those individuals who may not fully embrace astrosociology but can recognize its interesting possibilities. Contradictions abound capped with a final sentence that admits to the student's mixed sentiments.

The final two respondents were placed into the category of answering the final exam question as "not interested" in astrosociology and thus differ from those above in a fundamental way. They do not express mixed feelings about astrosociology. Instead, space exploration is viewed as an impractical undertaking or the respondent possesses no interest in learning about it. While the first student below identifies the potential value of astrosociology, it is not 
important enough to pursue in a new course. Sending people into outer space is seen as too dangerous, and apparently so is studying it is as well, although sending machines is acceptable.

Astrosociology is something I lack interest in. I don't consider space as a possible place for human societies to exist. I mean, sure, they can build satellites and other more technological devices there, but space is a lot different than Earth. I think that giving people more knowledge on what is beyond this Earth does change people's opinion about this issue but research does not provide sufficient information to tell us possible outcomes if societies were to exist out there. Space exploration in my opinion is something people would like to learn about but not something they want to get involved with and something they want to bet their lives on.

The final respondent below states that he or she benefitted from the Introduction to Sociology class. It covered traditional issues unrelated to outer space. However, he or she expresses no interest for astrosociology whatsoever in the response below - an uncharacteristically emphatic "not interested."

I don't think I would be interested because I am not really interested in learning about space exploration, although learning about sociology and the society around us has been a real good experience. I'm a new student straight out of High School and college has been a struggle for me. But I've learned so much from your lectures and videos. Thank you...

Bringing space into the classroom will not inspire all students to pursue careers in STEM subjects or astrosociology. For the student above, sociology was interesting enough without space exploration. Yet the exposure to NASA programs, shown in Figure 2, indicates an increased interest in space exploration. It did not imply that even most students would pursue STEM subjects due to an exposure to space exploration. Just the same, there does seem to be a significant effect that provides incentive to pursue these NASA programs.

3. Summary of Final Exam Responses

Similarly, if these community college students are any indication, then it seems as though astrosociology may very well represent a new field with great potential. Based on the examples of responses above, students express an interest in astrosociology even if they would not, or could not, personally take an Introduction to Astrosociology course. Even with the extremely limited sample compared to the entire population of college/university of students, this preliminary investigation bodes well for the argument that a fair number of students would indeed seek to become astrosociologists.

An important point to remember is that there is no point of reference for these students beyond what is provided in the final exam item. Just imagine how they would respond if an avenue to pursue astrosociology actually existed beyond the community college they attended. A most important goal to ensure the continued development of astrosociology will inevitably involve the creation of a new generation of formally-trained astrosociologists. These survey results, along with the author's personal experiences with students who express a strong interest in pursuing astrosociology, combine to provide a promising outlook for astrosociology in the academic world. $\$$

The preliminary research results of this pilot study indicate a strong interest to at least learn about astrosociology by taking an introductory class though no commitment to pursue it as a major or career. If such an interest proves viable in the entire student population at various levels of educational attainment, then bringing space into the social science classroom would likely motivate undeclared college students, for example, to explore the social sciences when they would otherwise be more inclined to consider other types of majors more strongly.

\section{To Cross a Threshold...}

Ultimately, the only way to develop astrosociology in a legitimate manner involves the creation of programs in existing departments of colleges and universities. The body of knowledge slowly accumulating since 2003 provides

\footnotetext{
$\S$ After reviewing the website Astrosociology.com, numerous students at various levels of attainment have contacted the author requesting how they could pursue astrosociology as a major in their sociology, psychology, and anthropology departments.
} 
prospective astrosociology majors with a sound grounding in the new field. ${ }^{* *}$ Unfortunately, they cannot pursue it at the present time due to its absence in the schools they attend. A few students have taken it upon themselves to instill change by beginning to pressure their professors and advisors to accept an astrosociology topic for theses and dissertations, though their success rate still remains inconclusive. Writing research papers about astrosociological topics in introduction and other courses may prove a more accessible way of seeding academia with astrosociology.

In order for astrosociology to truly become an academic field, the efforts of supporters must cross a threshold in which the preexisting (current) condition, an absence of astrosociology, is replaced by its formal presence in the academic world. It would take the form of either a substantial number of students demanding astrosociology courses or professors and other academicians doing the same. An even more effective strategy for astrosociology's development would consist of a "perfect storm" comprised of the simultaneous demand for this new field by both students and educators across the United States. In fact, the storm would be more "perfect" to the extent that all interested parties advocate it development. It would place enough pressures on departments around the country to begin considering astrosociology courses - perhaps starting with making available an Introduction to Astrosociology course on a trial basis. This would serve as a critical initial step. Once astrosociology's acceptance is demonstrated in academe, then the development of astrosociology will accelerate to new levels otherwise impossible. Similar gains at the high school level and below would also benefit its development immensely.

Once a particular department becomes bold enough to offer the first major in the field, it will herald the beginning of a new era in which the average sociologist/social scientist finally begins to regard the study of astrosocial phenomena as a much more serious matter. Since we cannot afford to presume such an outcome, it behooves supporters from all quarters to pursue the development of astrosociology. It is plausible that the combination of student and faculty pressures will result in the crossing of the threshold that finally results in the initial manifestations of formal astrosociology courses, followed later by the formation of astrosociology programs and departments. Space scientists, engineers, architects, and others in the space community who come to recognize how astrosociology can benefit their efforts serve as another source of support for the developing field and add to the effort further astrosociology's development as an academic field. Formal collaboration between the social science and space communities has always represented a vital goal from the outset of the author's efforts to develop this newly emerging field. This represents just another dimension of that mutual need to bring in the social-scientific perspective to the study of space exploration issues in both the non-academic and academic worlds.

\section{Conclusion}

Traditionally, sociology and the other social sciences have ignored astrosociological specializations for the most part - and certainly as comprising an area of concentration. Emphasis of astrosociology matters little if scholars in social science disciplines continue their traditional failure to study "space and society" issues. The core specializations listed in Table 2 are indeed quite relevant to the social sciences and they have much to offer! We must ask ourselves a simple question. What's wrong with the sociological discipline and the other social sciences? For fifty years, the space age has raged on without any great emphasis on space exploration and its relationship with social life and human behavior. Other subfields in sociology, for instance, such as deviance and racism, have garnered the most attention. Perhaps these disciplines are so entrenched in the protection of their positions that new subfields such as astrosociology face very difficult challenges to break into the existing structure.

In the next fifty years, it is likely that humanity will venture beyond its own backyard (i.e., the Earth-Moon system) ${ }^{19}$ and explore the solar system - even settle on Mars and establish space societies in other extraterrestrial environments - to an extent undreamed of before for many. In order to make this possible - and more importantly, successful - it will become necessary to bring in the social sciences in a big way. This requirement will require the formal education and training of astrosociologists. We had better get started introducing this new field into sociology, astronomy, and other social science departments and programs - or face the very real possibility of requiring knowledge regarding astrosocial phenomena with no one capable of providing it, or even of conducting research to attain it.

The ongoing development of astrosociology indicates that it has found a niche in the study of outer space that was missing for nearly the entire space age. In order for it to develop beyond the support of existing scientists, it must find its way into the curricula of educational organizations at the primary, secondary, and post-secondary levels. In other words, the need exists to educate and trains future generations of astrosociologists. This mandate is

\footnotetext{
** A growing number of astrosociology references exist on the Virtual Library page at Astrosociology.com. Many papers and articles were written by this author, but an increasing number come from various other contributors. See URL: http://www.astrosociology.com/vlibrary.html\#VL_ASRefs
} 
impossible at the current time due to the absence of astrosociology in our schools. It makes sense that the missing perspective $e^{20}$ in space research requires advocacy and a social movement to change perceptions and practical allocation of resources to support astrosociology as this new field threatens traditional subject areas within sociology and other disciplines.

Space can potentially motivate students to stay in school, and pursue majors that often contribute to continuation of space exploration over the next generation. While STEM subjects receive the greatest attention in this regard for good reasons, including the operation of past space program issues, the future of space exploration will necessarily change when human beings venture into space to live, work, and play on permanent and sustained bases. If this assumption is indeed correct, then any particular society that desires to pursue the exploration of space would benefit itself most if it utilized space to motivate all students regardless of their current interests or intentions. We will need astrosociologists just as much as space scientists and engineers when humans leave the Earth, so NASA will find that it needs a well-rounded workforce comprised of experts in the natural sciences and in the social sciences to work together.

NASA does a fairly good job in motivating students to pursue STEM subjects. However, their effectiveness has limitations. For one thing, some students find the social sciences and humanities more interesting or simply fail to find excitement in STEM subjects without much exposure to an astrosociological approach within their social science programs. As an amendment to its current efforts, rather than one intended to shift natural science students to the social sciences, NASA should pursue the development of astrosociological education in conjunction with its efforts regarding the STEM subjects. It will be a great day when a "social studies" or sociology teacher launches into space for example. In other words, NASA should promote a greater understanding of space from all scientific perspectives. By adding the "A" to the end of the STEM acronym (i.e., STEMA), the focus broadens to include the social sciences which increasingly have something to contribute to space exploration and related issues. This approach should motivate space scientists and engineers while simultaneously adding social science majors who would finally have the option of choosing to study space in the form of astrosociology.

It is time to add a new dimension to the development of astrosociology. It currently lacks a pedagogical component that will prove invaluable in its growth. Astrosociology courses and programs must develop. This would allow for a rush of students and educators to embrace this new field on a much grander scale as its existence in these forms would indicate the legitimacy of astrosociology. While the development of astrosociology can indeed continue without taking this vital step, it will remain limited and ultimately fail due to an insufficient number of astrosociologists who dedicate themselves to this cause. Therefore, it behooves current supporters who are now students to pressure their professors to allow astrosociology into the social science classroom and those who are now professors/educators to advocate the creation of course and curricula in their own departments. In this way, the development of astrosociology will grow through the attraction of new students who will come to call themselves "astrosociologists." Based on the limited survey presented in this article, this possibility does not seem farfetched at all. In fact, it may well result as the inevitable outcome of the movement to develop this new field. The author predicts that the development of astrosociology as an academic field will become a vital part of the effort to advance the space exploration effort in the twenty-first century and thereafter. This prediction is based on the increasing importance of the human dimension (as well as its growing recognition) and all of the complications that this brings to the space exploration table - and, most directly, this prediction is based on the corresponding imperative to understand these astrosociological issues.

\section{References}

${ }^{1}$ Pass, J., "Educating Astrosociologists: The Need to Bring Outer Space into Social Science Classrooms,” Astrosociology.com Virtual Library, URL: http://www.astrosociology.com/Library/PDF/PSA2007_Educating\%20Astrosociologists.pdf, 2007 [cited 3 August 2007].

${ }^{2}$ Pass, J., "The Astrosociology of Space Colonies: Or the Social Construction of Societies in Space." Space Technology and Applications International Forum (STAIF) Conference Proceedings, Volume 813, Issue 1, pp. 1153-1161. Virtual Library, URL: http://www.astrosociology.com/Library/PDF/Submissons/STAIF_Astrosociology\%20of\%20Space\%20ColoniesPDF.pdf [cited 4 June 2007].

${ }^{3}$ Tough, Allen, "Positive Consequences of SETI Before Detection" Acta Astronautica, Vol. 42, No. 10-12, 1998, pp. 745748. [Note: original 1995 posting of article on internet is at following URL, http://www.ieti.org/articles/before.htm].

${ }^{4}$ Harrison, A. A., Spacefaring: The Human Dimension, University of California Press, Berkeley, 2001.

${ }^{5}$ See reference note 4 , p. xi.

${ }^{6}$ Butz, W. P., Kelly, T. K., Adamson, D. M., Bloom, G. A., Fossum, D., and Gross M., E., Will the Scientific and Technology Workforce Meet the Requirements of the Federal Government?, Rand, Santa Monica, CA, 2004. 
${ }^{7}$ Kuenzi, J. J., Matthews, C. M., and Mangan, B. F., Science, Technology, Engineering, and Mathematics (STEM) Education Issues and Legislative Options, Congressional Research Service/The Library of Congress, Washington, D.C., URL: http://www.fas.org/sgp/crs/misc/RL33434.pdf, 2006 [cited 9 July 2007].

${ }^{8}$ See reference note 7, p. 17.

${ }^{9}$ Bray, Diane M., "Evaluation and Feedback Systems” (PowerPoint Presentation), Office of Education, National Aeronautics and Space Administration, Washington, D.C., URL: http://www.hq.nasa.gov/hqiso9000/ppt/ qual_coun_brief_11_05_03_CodeN.ppt, slide 4, 2003 [cited 2 July 2007].

${ }^{10}$ See reference note 9 , slide 6.

${ }^{11}$ See reference note 9 , slide 11 .

${ }^{12}$ Pass, J., "Viewpoint: Astrosociology as the Missing Perspective," Astropolitics, Vol. 4, No.1, 2006, pp. 85-99, p. 91.

${ }^{13}$ Harrison, Albert A., "Overcoming the Image of Little Green Men: Astrosociology and SETI,” Astrosociology.com Virtual Library, URL: http://www.astrosociology.com/Library/PDF/Submissons/Overcoming\%20LGM_Harrison.pdf, 2005 [cited 10 August 2007].

${ }^{14}$ Pass, J., “Applied Astrosociology: The New Imperative to Protect the Earth and Human Societies, Astrosociology.com Virtual Library, URL: http://www.astrosociology.com/Library/PDF/Protecting\%20Societies.pdf, 2006 [cited 11 July 2007].

${ }^{15}$ See reference note $11, \mathrm{p} 86$.

${ }^{16}$ See reference note 1 .

${ }^{17}$ Pass, J. "Developing Astrosociology for the Space Sciences," Astrosociology.com Virtual Library, URL: http://www.astrosociology.com/Library/PDF/Submissons/Developing\%20Astrosociology.pdf, 2006, p. 24 [cited 20 June 2007 ].

${ }^{18}$ Harrison, A. A., After Contact: The Human Response to Extraterrestrial Life, Plenum, New York, 1997.

${ }^{19}$ See reference note 17.

${ }^{20}$ See reference note 12 . 